\title{
Dark matter interpretation of the origin of non-thermal phenomena in galaxy clusters
}

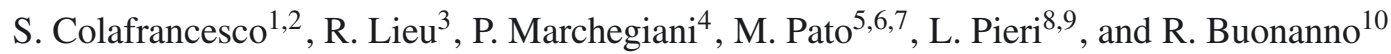 \\ 1 INAF - OAR via Frascati 33, 00040 Monteporzio, Italy \\ e-mail: sergio.colafrancesco@oa-roma.inaf.it \\ 2 on leave from: ASI viale Liegi 26, 00100 Roma, Italy \\ 3 The University of Alabama in Huntsville, Optics Building, Room 201 F, Huntsville, AL 35899, USA \\ e-mail: richardlieuuah@gmail.com \\ 4 Dipartimento di Fisica, Università di Roma La Sapienza, P.le A. Moro 2, Roma, Italy \\ e-mail: paolo.marchegiani@oa-roma.inaf.it \\ 5 Dipartimento di Fisica, Università degli Studi di Padova, via Marzolo 8, 35131 Padova, Italy \\ ${ }^{6}$ Institut d'Astrophysique de Paris, 98bis bd Arago, 75014 Paris, France \\ 7 Université Paris Diderot-Paris 7, rue Alice Domon et Léonie Duquet 10, 75205 Paris, France \\ e-mail: pato@iap.fr \\ 8 Istituto Nazionale di Fisica Nucleare, Sezione di Padova, via Marzolo 8, 35131 Padova, Italy \\ e-mail: lidia.pieri@gmail.com \\ 9 Dipartimento di Astronomia, Università degli Studi di Padova, Vicolo dell’Osservatorio 3, 35122 Padova, Italy \\ 10 Dipartimento di Fisica, Università di Roma Tor Vergata, Roma, Italy \\ e-mail: roberto.buonanno@roma2.infn.it
}

Received 6 April 2010 / Accepted 17 October 2010

ABSTRACT

\begin{abstract}
Aims. We studied the multi-frequency predictions of various annihilating dark matter (DM) scenarios in order to explore the possibility to interpret the still unknown origin of non-thermal phenomena in galaxy clusters.

Methods. We consider three different DM models with light $(9 \mathrm{GeV})$, intermediate $(60 \mathrm{GeV})$, and high $(500 \mathrm{GeV})$ neutralino mass and study their physical effects in the atmosphere of the Coma cluster. The secondary particles created in the neutralino annihilation processes produce a multi-frequency spectral energy distribution (SED) of non-thermal radiation and also heat the intracluster gas, which we test against the observations available for the Coma cluster from radio to gamma-rays. The various DM-produced SEDs are normalized by the condition to fit the Coma radio halo spectrum, thus obtaining best-fit values of the annihilation cross-section $\sigma \mathrm{V}$ and of the central magnetic field $B_{0}$.

Results. We find that it is not possible to interpret all the non-thermal phenomena observed in galaxy clusters in terms of DM annihilation. The light-mass DM model with $9 \mathrm{GeV}$ mass produces too little power at all other frequencies, while the high-mass DM model with $500 \mathrm{GeV}$ produces a large excess power at all other frequencies. The intermediate-mass DM model with $60 \mathrm{GeV}$ and $\tau^{ \pm}$composition is marginally consistent with the HXR and gamma-ray observations, but narrowly fails to reproduce the EUV and soft X-ray observations. The intermediate-mass DM model with $60 \mathrm{GeV}$ and $b \bar{b}$ composition is, on the other hand, always below the observed fluxes. We note that the radio halo spectrum of Coma is well fitted only in the $b \bar{b}$ or light- and intermediate-mass DM models. We also find that the heating produced by the DM annihilation in the centre of the Coma cluster is always larger than the intracluster gas cooling rate for an NFW DM density profile and it is substantially smaller than the cooling rate only for a cored DM density profile in light-mass DM model with $9 \mathrm{GeV}$.

Conclusions. The possibility of interpreting the origin of non-thermal phenomena in galaxy clusters with DM annihilation scenarios requires a low neutralino mass and a cored DM density profile. If we then consider the multi-messenger constraints to the neutralino annihilation cross-section, it turns out that this scenario would also be excluded unless we introduce a substantial boost factor that represents DM substructures. If we relax the condition to fit the Coma radio halo and we consider the EUV and HXR detections as upper limits for the non-thermal emission, together with the gamma-ray limit, then the limits on $\sigma V$ are less stringent than those obtained by the multi-messenger analysis.
\end{abstract}

Key words. cosmology: theory - dark matter - galaxies: clusters: general - galaxies: clusters: individual: Coma

\section{Introduction}

Observational evidence for the existence of diffuse components of non-thermal origin in clusters of galaxies and their wider implications is a topic of sustained debate among researchers. In particular, the presence of giant radio halos and relics (see Feretti \& Giovannini 2008, for a review) indicates that diffuse distributions of relativistic electrons and magnetic fields must exist in many clusters of galaxies. Moreover, some clusters also show "unusual" diffuse components, i.e. the excess soft and hard $\mathrm{X}$-ray emission above the level expected from the hot virialized cluster (e.g., Lieu et al. 1996; Fusco-Femiano et al. 1999; Kaastra et al. 1999), even if the observations are sometimes controversial (see, e.g., discussion in Rossetti \& Molendi 2004).

While the diffuse radio sources in galaxy clusters are universally interpreted as produced by synchrotron emission, the extreme ultraviolet (EUV) and hard X-ray (HXR) excesses have 
been interpreted as the combined effect of an underlying inverseCompton scattering (ICS) of the cosmic microwave background (CMB) against cluster relativistic electrons that complement the radio halo population (e.g., Hwang 1997; Ensslin \& Biermann 1998; Sarazin \& Lieu 1998; Blasi \& Colafrancesco 1999; Colafrancesco et al. 2007; Marchegiani et al. 2007; Colafrancesco \& Marchegiani 2009; Buote 2001), or thermal emission (Mittaz et al. 1998; Cen \& Ostriker 1999) from "missing baryons" at sub-virial temperatures (see, e.g., the review of Durret et al. 2008). Focusing upon the former interpretation, the role and ramifications of an underlying power law spectrum across the entire X-ray frequency band can be far reaching, with issues concerning cluster merging and central cooling, and also mass and spectroscopic consequences being addressed by Million \& Allen (2009), Lagana et al. (2010), and Prokhorov (2009). In the even broader context, the observed cosmic ${ }^{6} \mathrm{Li}$ abundance could be evidence for excess entropy in clusters (Nath et al. 2006), along with the surprisingly low level of Sunyaev-Zel'dovich effect in the Wilkinson Microwave Anisotropy Probe (WMAP) and South Pole Telescope (SPT) sample of many clusters (Diego and Partridge 2010; Lieu et al. 2006; Komatsu et al. 2010; Lueker et al. 2009), and the effects of the magnetic field on the intracluster gas distribution (e.g. Colafrancesco \& Giordano 2007) as a possible indication of significant intracluster non-thermal pressure.

Historically however, there have been numerous suggestions of a general, non-thermal intracluster environment, early examples are given in Jaffe (1977) and Rephaeli (1979) who considered Alfvén wave excitation. In particular, a model of the acceleration via Alfven waves driven by major cluster mergers is given in Brunetti et al. (2004), including the idea that relativistic jets carry a significant portion of the total energy output of radio galaxies, causing X-ray emission up to Mpc distance scales (Ghisellini \& Celotti 2001). Celotti et al. (2001) provided an analysis of such a scenario for PKS 0637-752.

A relevant, related observation (see Giovannini et al. 1993) maps the spectral index of cluster radio halos, and specifically of the Coma radio halo from $327 \mathrm{MHz}$ to $1.4 \mathrm{GHz}$. Here, we note the need for non-thermal emission by electrons with energy just above the energy range required by the non-thermal electron population that is supposed to produce soft and hard X-rays by ICS (see Lieu et al. 1999).

An important issue connected with the presence of diffuse emission of non-thermal origin in galaxy clusters is that relativistic electrons with $E \sim \mathrm{GeV}$ (as those which produce the radio halos) lose their energy in relatively short time by effect of synchrotron and ICS with the CMB photons interactions, and they should reach a short distance in comparison to the dimension of the halos (see discussion in Blasi \& Colafrancesco 1999).

Therefore, several solutions have been proposed to solve this problem, as the re-acceleration in situ of the electrons by Alfven wave excitation driven by major cluster mergers and induced turbulences (e.g., Jaffe 1977; Rephaeli 1979; Brunetti et al. 2004) or the production of secondary electrons in the interactions between non-thermal and thermal protons (e.g., Dennison 1980; Blasi \& Colafrancesco 1999; Marchegiani et al. 2007; Colafrancesco \& Marchegiani 2008).

The separate physical mechanism to be invoked is neutralino dark matter (DM) annihilation as a cluster's non-thermal reservoir (see Colafrancesco \& Mele 2001; Colafranceso et al. 2006) to compensate for losses of the particles as they are distributed throughout the cluster medium during the long process of particle diffusion (see, e.g., Völk et al. 1996; Colafrancesco \& Blasi 1998).
Today, several astrophysical evidences (e.g., gravitational lensing, galaxy rotation curves, galaxy clusters masses) indicate that most of the matter content of the universe is in form of DM, whose nature is still elusive. Several candidates have been proposed as fundamental DM constituents, ranging from axions to light, MeV DM, from Kaluza-Klein (KK) particles, branons, primordial black holes $(\mathrm{PBH})$, mirror matter to supersymmetric weakly interacting massive particles (WIMPs; see, e.g., Baltz 2004; Bertone et al. 2005; Bergström 2000, for reviews). In this paper we assume that the main DM constituent is the lightest neutralino of the minimal supersymmetric extension of the Standard Model (MSSM). This assumption can be tested by observing the radiation emitted by the secondary products of the neutralino annihilation processes, whose features have been studied (e.g., Colafrancesco \& Mele 2001; Colafrancesco et al. 2006). As pointed out by Colafrancesco et al. (2006), the relevant physical properties that determine the features of the emitted radiation are the composition of the neutralino, its mass, and the value of the annihilation cross section.

In this paper we explore the consequences of a neutralino DM annihilation scenario on the non-thermal particle content of galaxy clusters over a multi-frequency range (from radio to gamma-ray and $\mathrm{TeV}$ energies), and in particular on one of the best studied clusters so far, the Coma cluster of galaxies, for which an extensive multi-frequency coverage has been accumulated.

To this aim, we focus on three neutralino DM models with mass $M_{\chi}$ of 9,60 and $500 \mathrm{GeV}$.

Neutralinos with 60 and $500 \mathrm{GeV}$ mass are representative of respectively intermediate - and high-mass supersymmetric models that are consistent with accelerator and multi-messenger constraints, and it is also interesting to be probe them by direct detection experiments (like e.g. the recent CDMS results, see Ahmed et al. 2009). In this context, the $60 \mathrm{GeV}$ neutralino mass is close to the lower limit of the combination of the CDMS II experiment with the allowed parameter space calculated from various Minimal Supersymmetric Models (e.g., Roszkowski et al. 2007; Ellis et al. 2005), and it is interesting to possibly test this by a multi-frequency search limited to the gamma-ray energies probed by Fermi. On the other hand, it is interesting to test the $500 \mathrm{GeV}$ mass model by a multi-frequency search extended even in Cherenkov experiments realm.

The light-mass neutralino model with $M_{\chi}=9 \mathrm{GeV}$ has been recently worked out as a consistent solution of the CoGeNT and DAMA observations (Fitzpatrick et al. 2010). This low-mass neutralino is allowed in supersymmetric scenarios where the hypothesis of gaugino masses universality at the unification scale is released (Bottino et al. 2003).

To explore the astrophysical consequences of these DM models, we consider pure annihilation channels in order to highlight their observational features. We focus, specifically, on $b \bar{b}$ and $\tau^{ \pm}$annihilations for 9,60 and $500 \mathrm{GeV}$ mass neutralino models, and we also consider annihilations into $W^{ \pm}$for the $500 \mathrm{GeV}$ mass neutralino model. We leave the neutralino annihilation cross-section $\sigma V$ as a free parameter to be constrained by the astrophysical data in our analysis.

In this study, we also consider the constraints on the DM model and density profile coming from the heating rate of the thermal gas at the centre of the cluster.

The outline of the paper is the following. We describe in Sect. 2 the properties of the various neutralino DM models and the various DM density profiles that we consider for the Coma cluster. We derive the astrophysical consequences of the neutralino DM annihilation in Coma in Sect. 3 and focus on the 
Table 1. Density profiles for the Coma cluster. $\rho_{\mathrm{c}}=1.36 \times 10^{2} \quad M_{\odot} \mathrm{kpc}^{-3}$ is the critical density for closure of the universe.

\begin{tabular}{cccc}
\hline \hline Profile & $\alpha$ & $r_{\mathrm{s}}(\mathrm{kpc})$ & $\frac{\rho_{\mathrm{s}}}{\rho_{\mathrm{c}}}$ \\
\hline NFW & 1 & 287 & $1.96 \times 10^{4}$ \\
cored & 0 & 142 & $1.66 \times 10^{5}$ \\
NFW $^{N-\text { body }}$ & 1 & 450 & $6.75 \times 10^{3}$ \\
\hline
\end{tabular}

limits set on the expected DM-induced spectral energy distribution (SED) at multifrequency, and on the additional physical effects induced by DM annihilation. The discussion of our results and the conclusion of our analysis are presented in Sect. 4.

Throughout the paper, we use a flat, vacuum-dominated cosmological model with $\Omega_{\mathrm{m}}=0.3, \Omega_{\Lambda}=0.7$ and $H_{0}=$ $70 \mathrm{~km} \mathrm{~s}^{-1} \mathrm{Mpc}^{-1}$.

\section{The dark matter model}

A 9 or $60 \mathrm{GeV}$ supersymmetric particle is expected to annihilate in quarks and leptons. Direct annihilation into light fermions is helicity-suppressed, so that the main remaining channels of annihilations are $b \bar{b}$ and $\tau^{+} \tau^{-}$. Electrons and positrons will consequently be mainly produced through secondary processes of hadronization and/or decay. Direct annihilation into $\gamma \mathrm{s}$ happens at 1-loop level and is therefore highly suppressed too. The bulk of photons arises from the $\pi^{0} \rightarrow \gamma \gamma$ e.m. decay.

In addition to the aforementioned channels, a $500 \mathrm{GeV}$ supersymmetric particle can also annihilate into gauge bosons, heavy quarks, and Higgs particles. The yield of photons produced in these cases and their energy spectrum is very similar to each other, so that we choose as a representative case the annihilation into $W^{+} W^{-}$.

In this framework, we computed the positron/electron spectra with DarkSUSY (Gondolo et al. 2004) and the yield of gamma-ray photons according to Fornengo et al. (2004).

\subsection{The dark matter profile of the Coma cluster}

Recent determination of the mass and density profile of Coma include methods based on weak lensing (Kubo et al. 2007) and on the study of the velocity moments of early-type galaxies in the Coma cluster (Lokas \& Mamon 2003) or on the analysis of infall patterns around Coma (Geller et al. 1999). Numerical simulations also give a hint on the DM density profile inside clusters (Bullock et al. 2001). The results of astronomical measurements and numerical experiments lead to a determination of the mass profile that is consistent among the various methods within the errors and the spatial resolution of each approach. In this paper we use the results of Lokas \& Mamon (2003), since they provide a fit to the data with both a spiky NFW profile and a cored profile.

The total inferred dark matter mass of Coma is $M_{v i r}=1.2 \times$ $10^{15} M_{\odot}$ within a dark matter virial radius of $2.7 \mathrm{Mpc}$. The dark matter profile is parameterized as

$$
\rho(r)=\frac{\rho_{\mathrm{s}}}{\left(\frac{r}{r_{\mathrm{s}}}\right)^{\alpha}\left(1+\frac{r}{r_{\mathrm{s}}}\right)^{3-\alpha}} .
$$

Scale parameters and inner slope can be found in Table 1. Also shown are the parameters for an NFW profile as inferred by $N$-body simulations (Bullock et al. 2001).

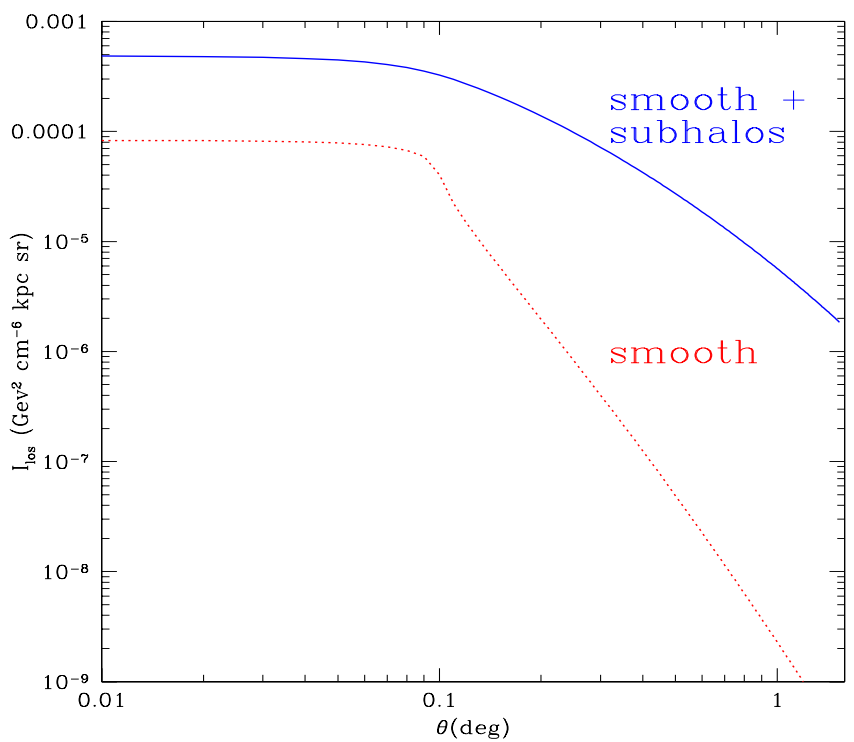

Fig. 1. Line of sight integral of the DM density squared is shown as a function of the projected angle from the cluster centre for a resolution of 0.1 degree (corresponding to a solid angle of $10^{-5} \mathrm{sr}$ ). The red dotted curve is for a smooth DM density profile while the blue solid curve is for a smooth DM density profile with the addition of subhalos (see text for details).

We also derive the effective boost factor computed as a line of sight (los) integral and taking into account the subhalos orbiting in the gravitational potential of the Coma cluster. For this calculation we follow the methodology and results of Pieri et al. (2009) in the framework suggested by the Via Lactea II numerical simulation (Diemand et al. 2008). We therefore make the assumptions that

i) the slope of the subhalo mass function is -2 , which implies for a DM halo such as Coma that about $64 \%$ of its mass is virialized in subhalos;

ii) the mass of subhalos is in the range $10^{-6} M_{\odot}-10^{-2} M_{\text {vir }}$;

iii) the radial dependence of the subhalo concentration parameter;

iv) an NFW profile for each of the subhalos.

We then compute the los integral with and without subhalos of the DM density squared to infer the boost factor $\mathcal{B}$ defined as the integral along the los and on the solid angle of the neutralino density squared in the case where we consider a smooth DM density profile or the sum of smooth DM density plus DM subhalos (see, e.g., Pieri et al. 2009). Fitting formulas for the concentration parameter as well as explicit notation for the line of sight integral including a population of subhalos can be found in Pieri et al. (2009). Figure 1 shows the result of our computation for an angular resolution $\Delta \Omega=10^{-5} \mathrm{sr}$ (corresponding to 0.1 degree). After integrating over the whole cluster volume, we obtain a boost factor $\mathcal{B}=35$. We do not expect significant deviation from this value when changing the subhalo density profile.

We stress that we consider subhalos down to a mass scale of $10^{-6} M_{\odot}$ because this is a fiducial value of the minimal subhalo mass for WIMPs (see, e.g., Green et al. 2005) even though values significantly lower or higher are also possible (Bringmann 2009). It is worth noticing that a lower value for the minimum subhalo mass would further increase the corresponding value of the integrated boost factor. 
In the following calculations we will first consider the case of a smooth DM density profile and then the effects of the boost factor.

\section{Astrophysical consequences}

We discuss here various astrophysical consequences of the DM models that we consider in this paper: electromagnetic (e.m.) signals (all of non-thermal origin) and heating of the intracluster gas induced by secondary particles produced in the DM annihilation processes.

The complete description of the emission features induced by $\mathrm{DM}$ annihilation is given by using the diffusion equation (i.e. neglecting convection and re-acceleration effects) given in Colafrancesco et al. (2006, see their Eq. (35)) which consistently takes into account the diffusion and energy loss properties of the secondary particles produced in the neutralino DM annihilation process.

The energy loss term is the sum of the effects due to inverse Compton, synchrotron radiation, Coulomb losses and bremsstrahlung (see Eq. (A.16) in Colafrancesco et al. 2006, for details). For electron energies $\gtrsim \mathrm{GeV}$ the inverse Compton and synchrotron terms dominate the losses, while for energies below $\sim 140 \mathrm{MeV}$, Coulomb losses dominate (see Fig. A.3 in Colafrancesco et al. 2006). For a more detailed description of the energy losses in the Coma cluster, we use throughout the paper a B-field radial profile $B(r) \propto n_{\text {th }}(r)$, where $n_{\text {th }}$ is the intracluster gas number density, unless otherwise specified.

The derivation of the full solution of the diffusion equation and the effects of diffusion and energy losses are given in Colafrancesco et al. (2006, see Eq. (A.1)) and we refer the reader to this paper for technical details. We consider here the same analytical approach and present the results of the multi-frequency emission produced by the DM models under study for the Coma cluster. We also compute the DM-induced signals in the limit in which electrons and positrons lose energy on a timescale much shorter than the timescale for spatial diffusion, i.e. the regime that applies to galaxy clusters (see Colafrancesco et al. 2006, for a discussion).

The part of the SED from which the strongest spectral constraints can be set is the low-frequency radio band from $\sim 30 \mathrm{MHz}$ to $\sim 5 \mathrm{GHz}$. Figure 2 shows the spectra of the diffuse radio emission of Coma calculated in the various DM models that we consider here. The best-fit values of the central value of the magnetic field $B_{0}$ and $\sigma V$ have been obtained from the fit to the Coma radio halo spectrum and normalizing the curves at the data point at $608 \mathrm{MHz}$.

We notice that the radio-halo spectrum could distinguish between the different neutralino compositions, preferring a $b \bar{b}$ composition because of its better-fitting intrinsic spectral curvature (see Fig. 2).

The fit is influenced by the value of the B-field. Changing the intensity of the B-field implies to change the energies of the electrons that emit by synchrotron in this frequency range, and this implies, in turn, that the resulting radio halo spectrum has a change in the spectral shape according to the value of $B_{0}$. These effects are illustrated in Fig. 2. For $M_{\chi}=9 \mathrm{GeV}$, the $b \bar{b}$ model recovers quite well the curvature of the observed spectrum for $B_{0}=20 \mu \mathrm{G}$, while the $\tau^{ \pm}$model reproduces the observed spectrum for a value of $B_{0}=2.2 \mu \mathrm{G}$. For $M_{\chi}=60 \mathrm{GeV}$, the $b \bar{b}$ model recovers quite well the curvature of the observed spectrum for $B_{0}=0.5 \mu \mathrm{G}$, while the $\tau^{ \pm}$model reproduces the observed spectrum only for the frequency range $v>100 \mathrm{MHz}$ for a value of $B_{0}=0.1 \mu \mathrm{G}$. For $M_{\chi}=500 \mathrm{GeV}$, the $b \bar{b}$ model reproduces
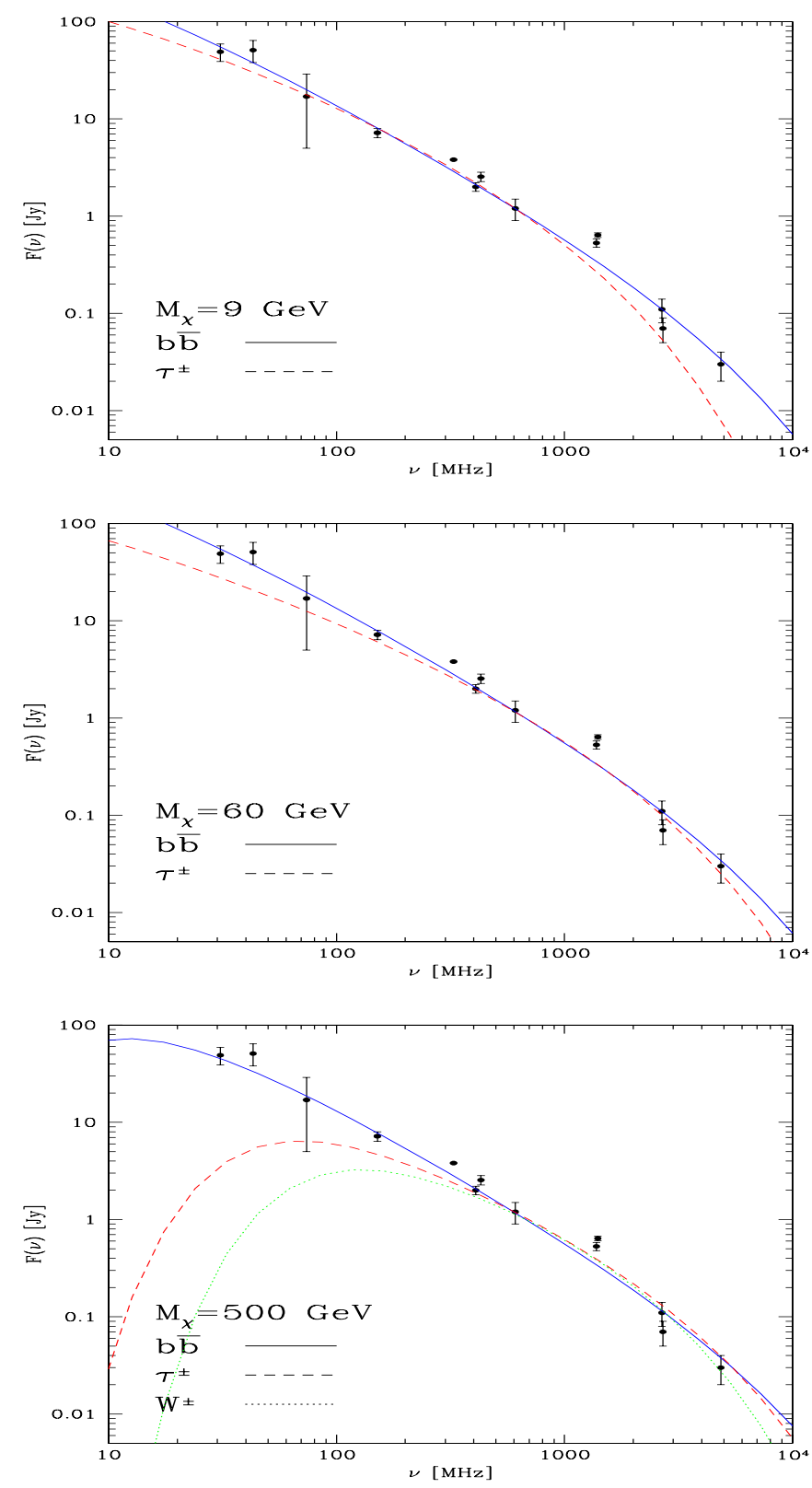

Fig. 2. Radio halo spectrum of Coma and the best fits of three DM models with $M_{\chi}=9 \mathrm{GeV}$ (upper panel) for $b \bar{b}$ (solid with $\sigma V=$ $1.7 \times 10^{-25} \mathrm{~cm}^{3} \mathrm{~s}^{-1}$ and $B_{0}=20 \mu \mathrm{G}$ ) and $\tau^{ \pm}$(dashed with $\sigma V=$ $5.6 \times 10^{-25} \mathrm{~cm}^{3} \mathrm{~s}^{-1}$ and $B_{0}=2.2 \mu \mathrm{G}$ ), $M_{\chi}=60 \mathrm{GeV}$ (mid panel) for $b \bar{b}$ (solid with $\sigma V=8.5 \times 10^{-23} \mathrm{~cm}^{3} \mathrm{~s}^{-1}$ and $B_{0}=0.5 \mu \mathrm{G}$ ) and $\tau^{ \pm}$(dashed with $\sigma V=9.0 \times 10^{-22} \mathrm{~cm}^{3} \mathrm{~s}^{-1}$ and $B_{0}=0.1 \mu \mathrm{G}$ ), and with $M_{\chi}=$ $500 \mathrm{GeV}$ (lower panel) with $b \bar{b}$ (solid with $\sigma V=2.0 \times 10^{-18} \mathrm{~cm}^{3} \mathrm{~s}^{-1}$ and $\left.B_{0}=0.01 \mu \mathrm{G}\right), \tau^{ \pm}$(dashed with $\sigma V=1.9 \times 10^{-17} \mathrm{~cm}^{3} \mathrm{~s}^{-1}$ and $B_{0}=0.002 \mu \mathrm{G}$ ) and $\mathrm{W}^{ \pm}$(dotted with $\sigma V=1.3 \times 10^{-16} \mathrm{~cm}^{3} \mathrm{~s}^{-1}$ and $B_{0}=0.001 \mu \mathrm{G}$ ). Data from Thierbach et al. (2003). The results are obtained using $B(r) \propto n_{\mathrm{th}}(r)$ and NFW smooth DM profiles, without considering the effect of substructures.

the curvature of the observed spectrum for $B_{0}=0.01 \mu \mathrm{G}$, while the $\tau^{ \pm}$and $W^{ \pm}$models require values of the central magnetic field that are much lower $\left(B_{0}=0.002-0.001 \mu \mathrm{G}\right)$ and can reproduce the spectral shape of the radio halo only for $v>400 \mathrm{MHz}$. The corresponding best-fit values of the annihilation cross section $\sigma V$ are given in the caption of Fig. 2.

Figure 3 shows the radio halo spectrum calculated in the various DM models that we consider here where we assumed the 


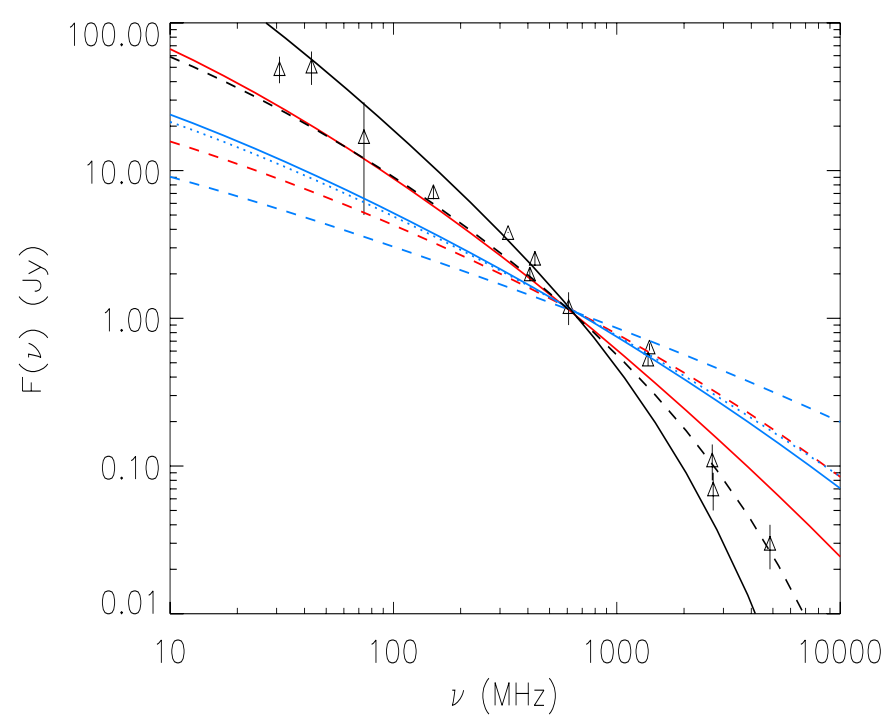

Fig. 3. Radio halo spectrum of Coma and the predictions of DM models calculated by assuming $B_{0}=4.7 \mu \mathrm{G}$ and $B(r) \propto n_{\mathrm{th}}(r)^{0.5}$; black lines refer to $M_{\chi}=9 \mathrm{GeV}$ with $b \bar{b}$ (solid line; $\sigma V=6.0 \times 10^{-25} \mathrm{~cm}^{3}$ $\mathrm{s}^{-1}$ ) and $\tau^{ \pm}$(dashed line; $\sigma V=1.4 \times 10^{-25} \mathrm{~cm}^{3} \mathrm{~s}^{-1}$ ); red lines refer to $M_{\chi}=60 \mathrm{GeV}$ with $b \bar{b}$ (solid line; $\sigma V=1.0 \times 10^{-24} \mathrm{~cm}^{3} \mathrm{~s}^{-1}$ ) and $\tau^{ \pm}$(dashed line; $\sigma V=9.0 \times 10^{-25} \mathrm{~cm}^{3} \mathrm{~s}^{-1}$ ); blue lines refer to $M_{\chi}=500 \mathrm{GeV}$ with $b \bar{b}$ (solid line; $\sigma V=7.5 \times 10^{-24} \mathrm{~cm}^{3} \mathrm{~s}^{-1}$ ), $\tau^{ \pm}$(dashed line; $\sigma V=3.0 \times 10^{-23} \mathrm{~cm}^{3} \mathrm{~s}^{-1}$ ) and $W^{ \pm}$(dotted line; $\left.\sigma V=1.1 \times 10^{-23} \mathrm{~cm}^{3} \mathrm{~s}^{-1}\right)$. The results are obtained using NFW smooth DM profiles, without considering the effect of substructures.

value of the central magnetic field derived from the most recent analysis of Faraday rotation measures, $B_{0}=4.7 \mu \mathrm{G}$ with a radial profile given by $B(r) \propto n_{\mathrm{th}}(r)^{0.5}$ (Bonafede et al. 2010). In this figure we fixed the value of $\sigma V$ in a way that the radio halo spectrum fits the observed one at $608 \mathrm{MHz}$. For this value of the central B-field, the DM model with $M_{\chi}=9 \mathrm{GeV}$ and $\tau^{ \pm}$composition and the model with $M_{\chi}=60 \mathrm{GeV}$ and $b \bar{b}$ composition can (marginally) reproduce the observed shape of the spectrum, while the model with $9 \mathrm{GeV}$ and $b \bar{b}$ composition is too steep and all other DM models provide radio halo spectra much flatter (harder) than the measured one.

The observed radial profile of the surface brightness of the Coma radio halo (see, e.g., Deiss et al. 1997) provides further astrophysical constraints. We find indeed that any DM profile, both NFW or cored, produces a too steep radio brightness profile that cannot reproduce the observed radio brightness profile if we consider radial profiles of the magnetic field decreasing towards the outer regions of the cluster, consistent with Faraday rotation measures (see, e.g., Kim et al. 1990; Bonafede et al. 2010). We find that the radial profile of the radio brigthness produced by the DM annihilation is compatible with the observed one only if a magnetic field radial profile that increases with the radius, as $B(r) \propto n_{\text {th }}(r)^{-3}$, is assumed, or if the DM subhalos are distributed in a way that the overall DM radial profile is less steep than the NFW or cored ones. This result is analogous to that found by Colafrancesco et al. (2006).

The overall SEDs of the DM models derived from the fits to the Coma radio halo spectrum populate the high-frequency regions of the e.m. spectrum differently. The SEDs of a $9 \mathrm{GeV}$, of a $60 \mathrm{GeV}$ and of a $500 \mathrm{GeV}$ neutralino model annihilating in Coma are shown in Fig. 4.
The 9 and $60 \mathrm{GeV}$ mass DM models that best fit the radiohalo spectrum of Coma produce ICS and bremsstrahlung radiation and $\pi^{0} \rightarrow \gamma \gamma$ radiation consistent with the existing limits at multi-frequency. Under the previous conditions, these signals particularly cannot be the nature of the soft and hard X-ray emission excesses detected in this cluster. Only the $60 \mathrm{GeV} \tau^{ \pm}$neutralino model can fit both the radio halo spectrum and the hard X-ray data, but not the Extreme UV Explorer (EUVE) data.

The DM model with $500 \mathrm{GeV}$ mass can reproduce the Coma radio halo spectrum only with very low values of the central magnetic field, $B_{0} \sim 0.001-0.01 \mu \mathrm{G}$. This has the consequence of requiring quite high values for the annihilation cross section $\sigma V \sim 10^{-18}-10^{-16} \mathrm{~cm}^{3} / \mathrm{s}$, and therefore for the secondary electron density produced by the neutralino annihilation. Thus, we obtain that the ICS emission expected from these models is very large and exceeds both the EUVE and BeppoSAX data as well as the EGRET and Fermi upper limits on Coma (the HESS upper limit on Coma at very high energies cannot strongly constrain the SED produced in the DM models that we consider here). We conclude, therefore, that neutralino DM models with mass of the order of $500 \mathrm{GeV}$ must be excluded by our analysis.

The shape of the SED does not change substantially when a cored DM profile is considered. The only effect is that the cored DM profile requires slightly higher values on $\sigma V$ to fit the Coma radio halo data.

We also evaluated the intracluster gas heating induced by DM annihilation (see Fig. 5) and found that an NFW DM density profile produces an excess heating in the inner $\sim 3 \mathrm{kpc}$ and $\sim 30 \mathrm{kpc}$ regions of Coma for 9 and $60 \mathrm{GeV}$, respectively. This effect provides therefore a strong constraint to the annihilation cross-section for these DM models. In order to achieve a lower DM-induced heating rate than the bremsstrahlung cooling rate at the cluster center, the annihilation cross-section worked out here must be reduced by a factor $\sim 10^{7}-10^{12}$.

The heating rate for the a cored DM profile with a mass of $500 \mathrm{GeV}$ is higher than the bremsstrahlung cooling rate of Coma for every DM annihilation channel. Therefore, this result also excludes the $500 \mathrm{GeV}$ mass neutralino model. DM models with a mass of $60 \mathrm{GeV}$ and a cored profile show, instead, a heating rate lower than the bremsstrahlung cooling rate for the $b \bar{b}$ composition, and are therefore consistent with both multi-frequency SED data and with heating rate constraints, while the $\tau^{ \pm}$model is not consistent because it has a heating rate higher than the cooling rate. For a neutralino mass of $9 \mathrm{GeV}$ and cored DM profile, both $b \bar{b}$ and $\tau^{ \pm}$models are consistent with heating rate constraints.

\section{Discussion and conclusions}

Any DM interpretation of astrophysical data has intrinsic multifrequency correlations and should, therefore, be tested against a series of independent observational constraints.

The full SED induced by DM annihilation in Coma indicates that the non-thermal e.m. signals induced by a 9 or $60 \mathrm{GeV}$ neutralino can be consistent with the shape of the radio-halo spectrum and produce ICS, bremsstrahlung radiation, and $\pi^{0} \rightarrow \gamma \gamma$ radiation consistent with the existing limits at multi-frequency. However, these signals particularly cannot be the nature of the soft and hard X-ray emission excesses detected in the Coma cluster. An exception to this point is given by the $\tau^{ \pm}$model with mass $60 \mathrm{GeV}$ that is able to fit the hard X-ray emission observed in Coma. However, in the framework of this model, the EUV excess in Coma should then be interpreted as owing to thermal emission from warm baryons at sub-virial temperature. 

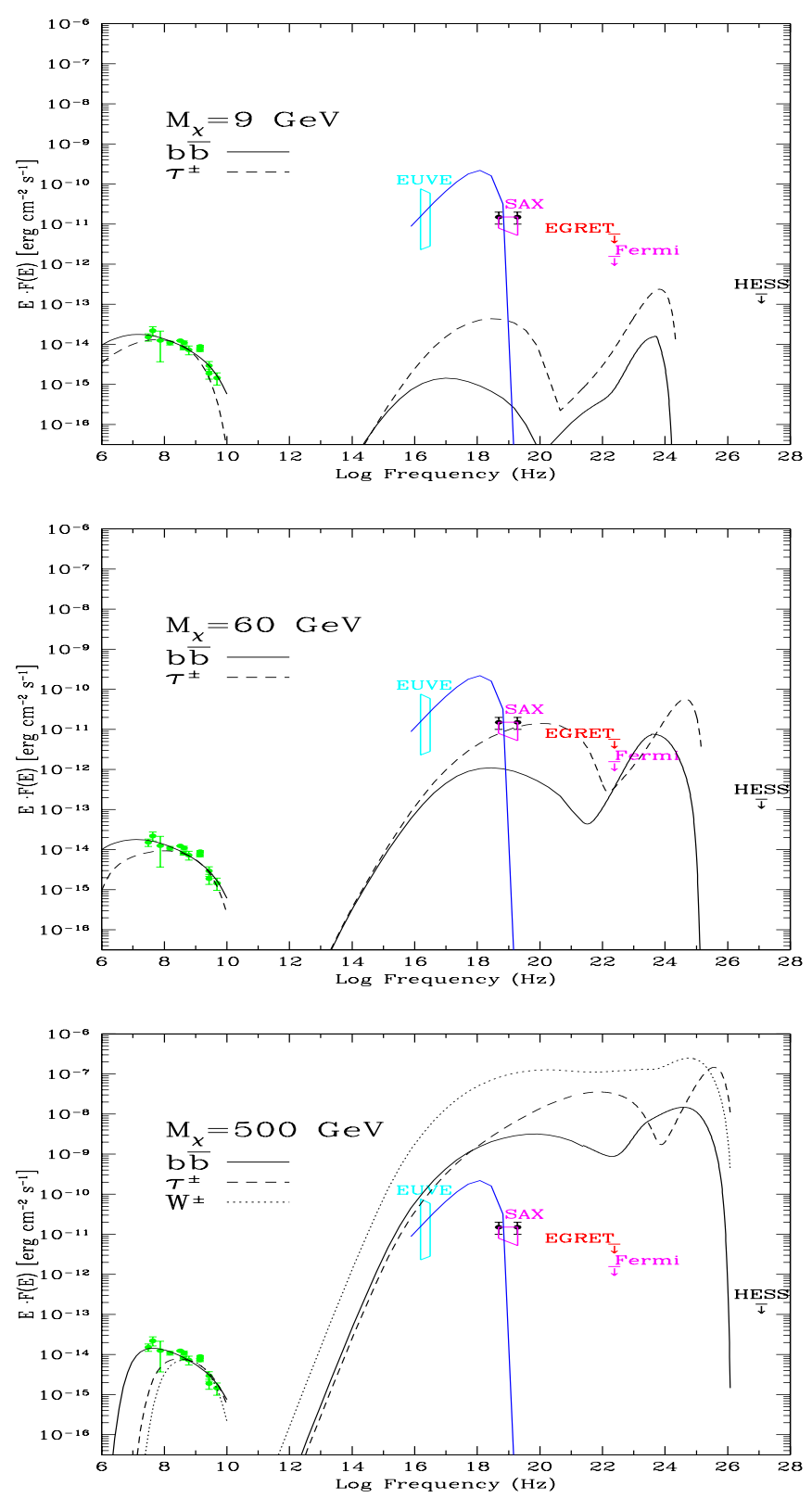

Fig. 4. SED of the DM-induced e.m. signals in Coma as predicted by the three DM models. Upper panel: $M_{\chi}=9 \mathrm{GeV}$ (solid: $b \bar{b}$; dashed: $\tau^{ \pm}$); mid panel: $M_{\chi}=60 \mathrm{GeV}$ (solid: $b \bar{b}$; dashed: $\tau^{ \pm}$); lower panel: $M_{\chi}=500 \mathrm{GeV}$ (solid: $b \bar{b}$; dashed: $\tau^{ \pm}$; dotted: $W^{ \pm}$) The values of $\sigma V$ and $B_{0}$ for the different models are the same as those used in Fig. 2. The hatched regions in the X-ray domain represent data from PDS/BeppoSAX (Fusco-Femiano et al. 2004), HEXTE/RXTE (Rephaeli et al. 1999) and EUVE (Lieu et al. 1999). We also report the EGRET (Reimer et al. 2003), Fermi (Mori 2009) and HESS (Aharonian et al. 2009a) upper limits on Coma. The blue line is a thermal bremsstrahlung model for Coma with $k T=8.2 \mathrm{keV}$ (Briel et al. 1992). The results are obtained using $B(r) \propto n_{\text {th }}(r)$ and NFW smooth DM profiles, without considering the effect of substructures.

Focusing on the radio frequency range,, we showed that a DM particle annihilating into $b \bar{b}$ best-fits the spectral shape of the radio halo of the Coma cluster quite independently of the neutralino mass. These models can also reproduce the Coma radio halo brightness profile only if the DM subhalo population is distributed in a way to make the overall DM radial distribution less steep than the NFW or cored DM profiles. However, the

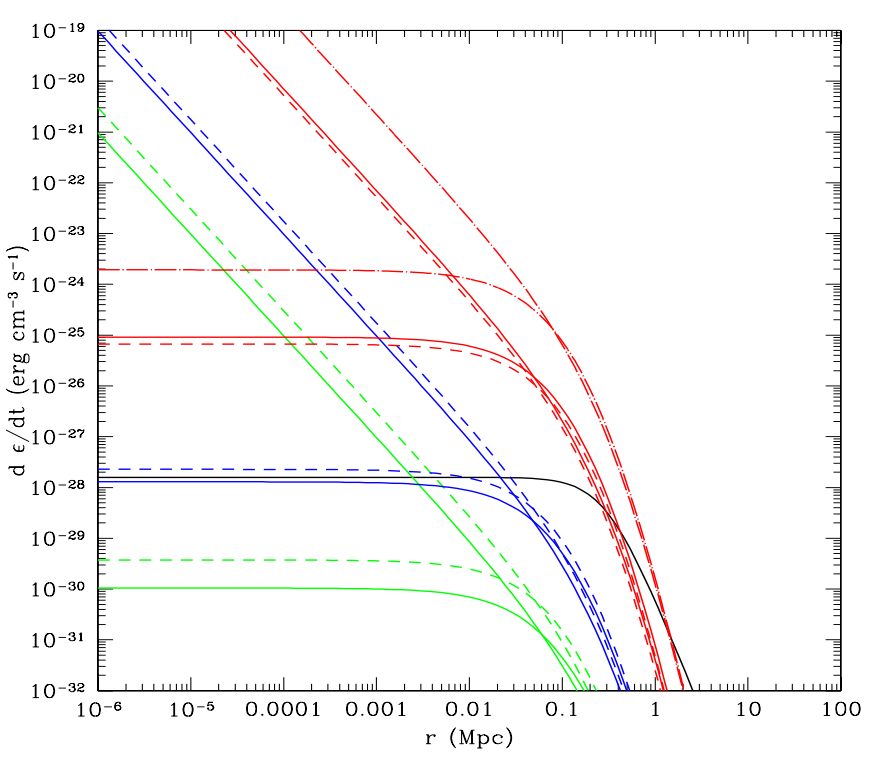

Fig. 5. Heating rate induced by DM secondary particles in Coma as predicted by the three DM models with $M_{\chi}=9 \mathrm{GeV}, M_{\chi}=60 \mathrm{GeV}$ and $M_{\chi}=500 \mathrm{GeV}$ for $b \bar{b}$ (solid), $\tau^{ \pm}$(dashed), $\mathrm{W}^{ \pm}$(dot-dashed) compositions. We consider an NFW DM profile (green, blue, and red peaking curves for 9, 60, and $500 \mathrm{GeV}$ respectively) and a cored DM profile (green, blue and red flattening curves for 9,60 , and $500 \mathrm{GeV}$ respectively). The values of $\sigma V$ and $B_{0}$ for the different models are the same as those used in Fig. 2. The solid black curve shows the bremsstrahlung cooling rate of the intra-cluster gas at a temperature of $8.2 \mathrm{keV}$. The results are obtained using $B(r) \propto n_{\text {th }}(r)$ and smooth DM profiles, without considering the effect of substructures.

radio halo spectrum fit requires that i) the value of the neutralino annihilation cross section $\sigma V$ strongly increases with increasing neutralino mass, and that; ii) the best-fit value of the central magnetic field in Coma decreases with increasing neutralino mass.

In the $9 \mathrm{GeV}$ case the value of the central magnetic field required in the $b \bar{b}$ case $\left(B_{0} \sim 20 \mu \mathrm{G}\right)$ is, however, too high compared to the results of Faraday rotation measures (Carilli \& Taylor 2002; Bonafede et al. 2010), while in the $\tau^{ \pm}$case an acceptable value of $B_{0} \sim 2.2 \mu \mathrm{G}$ is required. The best-fit values of $B_{0}$ decrease then to still marginally acceptable values $\sim 0.1-0.5 \mu \mathrm{G}$ for the $60 \mathrm{GeV}$ case (still consistent with the available observational limits, see Carilli \& Taylor 2002), while they take extreme values as low as $0.001-0.01 \mu \mathrm{G}$ for the highest neutralino mass (i.e. $500 \mathrm{GeV}$ ) we consider in our study.

We also studied the variation of $\sigma V$ for increasing values of $B_{0}$ that could still provide reasonable fit to the Coma radio halo spectrum. Figure 6 shows that the curves in the $\sigma V-B_{0}$ plane that are locus of the best fit to the Coma radio halo flatten to approximately the same value of $\sigma V$ for high values of $B_{0}$, i.e. when the energy losses are dominated by synchrotron losses.

The shaded bands in Fig. 6 encompass the range of upper limits on the annihilation cross-section derived from multi-messenger constraints. These constraints, summarized in Table 2, include positrons, antiprotons, radio emission, $\gamma$-rays from the Galactic Centre and the optical depth of CMB photons. Each column in Table 2 shows the limits derived using different galactic DM distributions: via Lactea II (VL2), Aquarius (Aq), and a cored isothermal profile (iso). Each cell features three values corresponding to the minimal, mean, and maximal propagation setups respectively. Also shown is the channel that produces the quoted upper limit - "rad", "pos", "anp", "GCg" and "opt" 
Table 2. The upper limits on the annihilation cross-sections from several measurements for the seven benchmark DM models.

\begin{tabular}{|c|c|c|c|c|}
\hline \multicolumn{5}{|c|}{ Upper limit on the annihilation cross-section $\left(10^{-26} \mathrm{~cm}^{3} \mathrm{~s}^{-1}\right)$} \\
\hline \multicolumn{2}{|c|}{ DM benchmark } & VL2 & $\mathrm{Aq}$ & Iso \\
\hline$M_{\chi}=9 \mathrm{GeV}$ & $\chi \chi \rightarrow \tau^{+} \tau^{-}$ & $(\mathbf{0 . 1}, \mathbf{0 . 1}, \mathbf{0 . 1})(\mathrm{rad}, \mathrm{rad}, \mathrm{rad})$ & $(\mathbf{7 . 8 , 7 . 8 , 7 . 8 )}(\mathrm{opt}, \mathrm{opt}, \mathrm{opt})$ & $(\mathbf{7 . 8 , 7 . 8 , 7 . 8 )}(\mathrm{opt}, \mathrm{opt}, \mathrm{opt})$ \\
\hline$M_{\chi}=9 \mathrm{GeV}$ & $\chi \chi \rightarrow b \bar{b}$ & $(0.06,0.06,0.06)(\mathrm{rad}, \mathrm{rad}, \mathrm{rad})$ & $(4.3,0.7,0.2)$ (opt,anp,anp) & $(4.3,2.6,0.8)(o p t, a n p, a n p)$ \\
\hline$M_{\chi}=60 \mathrm{GeV}$ & $\chi \chi \rightarrow \tau^{+} \tau^{-}$ & $(2.2,2.2,2.2)(\mathrm{rad}, \mathrm{rad}, \mathrm{rad})$ & $(5.3,3.6,2.1)$ (pos,pos,pos) & $(\mathbf{1 2}, 8.5,8.0)$ (pos,pos,pos) \\
\hline$M_{\chi}=60 \mathrm{GeV}$ & $\chi \chi \rightarrow b \bar{b}$ & $(0.6,0.6,0.6)(\mathrm{rad}, \mathrm{rad}, \mathrm{rad})$ & $(8.5,0.8, \mathbf{0 . 3})$ (anp,anp,anp) & $(\mathbf{1 9}, 3.8,1.7)$ (anp,anp,anp) \\
\hline$M_{\chi}=500 \mathrm{GeV}$ & $\chi \chi \rightarrow \tau^{+} \tau^{-}$ & $(106,101,73)$ (rad,pos,pos) & $(125,64,34)(\mathrm{GCg}, \mathrm{pos}, \mathrm{pos})$ & $(349,184,170)$ (pos,pos,pos) \\
\hline$M_{\chi}=500 \mathrm{GeV}$ & $\chi \chi \rightarrow W^{+} W^{-}$ & $(20,20,7.0)(\mathrm{rad}, \mathrm{rad}, \mathrm{anp})$ & $(74,8.6,3.5)(p o s, a n p, a n p)$ & $(\mathbf{1 6 2}, 46,20)$ (pos,anp,anp) \\
\hline$M_{\chi}=500 \mathrm{GeV}$ & $\chi \chi \rightarrow b \bar{b}$ & $(12,12,7.4)(\mathrm{rad}, \mathrm{rad}, \mathrm{anp})$ & $(54,9.2,3.7)($ pos,anp,anp $)$ & $(\mathbf{1 1 8}, 49,21)(\mathrm{pos}, \mathrm{anp}, \mathrm{anp})$ \\
\hline
\end{tabular}

Table 3. The upper limits on the annihilation cross-sections obtained for the seven benchmark DM models from the EUV, HXR and Fermi-LAT gamma-ray data.

\begin{tabular}{|c|c|c|c|c|}
\hline \multicolumn{5}{|c|}{ Upper limit on the annihilation cross-section $\left(\mathrm{cm}^{3} \mathrm{~s}^{-1}\right)$} \\
\hline \multicolumn{2}{|c|}{ DM benchmark } & \multirow{2}{*}{$\frac{\text { EUV }}{8.1 \times 10^{-22}}$} & \multirow{2}{*}{$\frac{\text { HXR }}{1.2 \times 10^{-22}}$} & \multirow{2}{*}{$\frac{\text { Gamma }}{1.9 \times 10^{-23}}$} \\
\hline$M_{\chi}=9 \mathrm{GeV}$ & $\chi \chi \rightarrow \tau^{+} \tau^{-}$ & & & \\
\hline$M_{\chi}=9 \mathrm{GeV}$ & $\chi \chi \rightarrow b \bar{b}$ & $3.7 \times 10^{-22}$ & $4.4 \times 10^{-22}$ & $4.9 \times 10^{-23}$ \\
\hline$M_{\chi}=60 \mathrm{GeV}$ & $\chi \chi \rightarrow \tau^{+} \tau^{-}$ & $2.6 \times 10^{-20}$ & $1.0 \times 10^{-21}$ & $6.3 \times 10^{-22}$ \\
\hline$M_{\chi}=60 \mathrm{GeV}$ & $\chi \chi \rightarrow b \bar{b}$ & $4.5 \times 10^{-21}$ & $9.3 \times 10^{-22}$ & $6.4 \times 10^{-23}$ \\
\hline$M_{\chi}=500 \mathrm{GeV}$ & $\chi \chi \rightarrow \tau^{+} \tau^{-}$ & $1.7 \times 10^{-18}$ & $3.9 \times 10^{-20}$ & $1.1 \times 10^{-21}$ \\
\hline$M_{\chi}=500 \mathrm{GeV}$ & $\chi \chi \rightarrow W^{+} W^{-}$ & $2.0 \times 10^{-19}$ & $1.1 \times 10^{-20}$ & $1.1 \times 10^{-21}$ \\
\hline$M_{\chi}=500 \mathrm{GeV}$ & $\chi \chi \rightarrow b \bar{b}$ & $1.2 \times 10^{-19}$ & $7.9 \times 10^{-21}$ & $1.1 \times 10^{-21}$ \\
\hline
\end{tabular}

stand for radio, positrons, antiprotons, Galactic Centre $\gamma$-rays, and CMB optical depth, respectively. The extreme values for each benchmark are indicated in bold and delimit the shaded regions of Fig. 6.

The uncertainty related to the propagation of antimatter in our Galaxy is taken into account by using minimal, mean, and maximal propagation setups as in Donato et al. (2004). Galactic DM distribution is modeled according to the smooth and clumpy distributions found in the latest high-resolution $N$-body simulations, namely Via Lactea II (Diemand et al. 2008) and Aquarius (Springel et al. 2008). As a conservative case, a cored isothermal profile with no substructure is also considered. For more details we refer to Pato et al. (2009), Pieri et al. (2009) and Catena et al. (2009). We stress that these multi-messenger bounds are quite robust and not easily avoidable, and therefore they set further constraints to the seven benchmark DM models studied in the present work (see Fig. 6). Other upper limits on the annihilation cross-section may be derived e.g. from the observation of dwarf galaxies with the Fermi satellite (Farnier 2009; see Fig. 3 of Abdo et al. 2010), which exclude values higher than $\sim 10^{-25} \mathrm{~cm}^{3} \mathrm{~s}^{-1}$ for a neutralino mass of $60 \mathrm{GeV}$. However, taking into account the uncertainty in the density profile and the dependence of the Fermi-LAT sensitivity from the assumed source spectrum (which is rather steep for the neutralino DM annihilation), such a limit could be released towards slightly higher values. The flattening of the curves in the $\sigma V-B_{0}$ plane for high values of $B_{0}$ means that it is not possible to have values of $\sigma \mathrm{V}$ that match the allowed region of annihilation cross sections suggested by multi-messenger studies of DM annihilation simply by increasing the value of the central magnetic field in galaxy clusters.

The lower set of curves in Fig. 6 shows the annihilation cross-sections required to fit the radio halo spectrum if DM substructures in Coma are considered (according to our discussion in Sect. 2). The effect of substructures is to increase the surface brightness intensity of the radiation emitted by the electrons, especially at large radii. As shown in Fig. 6, the inconsistency between fitting the radio halo and the multi-messenger analysis is significantly improved, particularly for $B_{0} \gtrsim 1 \mu \mathrm{G}$. However, none of the DM models under study is safely below the annihilation cross-section upper limits obtained from the multimessenger constraint analysis, whose results are summarized in Table 2.

If we relax the condition to fit the Coma radio halo data, constraints on the annihilation cross-section $\sigma V$ can be still obtained from the data on the diffuse non-thermal emission in Coma as derived in other spectral bands, i.e. the EUV, HXR, and gammaray energy bands. Since the physical mechanism responsible for the excess EUV and HXR emissions from the cluster are still not well understood, we conservatively consider their fluxes as upper limits for the dark matter annihilation radiation as those in the gamma-ray band. Table 3 reports the upper limits on $\sigma V$ for the benchmark neutralino models we consider when only the EUV, HXR, and Fermi-LAT gamma-ray limits are taken into account. Each column of Table 3 shows the limits derived using different bands measurements: EUV from Lieu et al. (1999), HXR from Fusco-Femiano et al. (2004), Fermi-LAT gamma-ray from Mori (2009). We note that these last upper limits are less stringent than those obtained from the multi-messenger analysis (see Table 2), and that they are compatible with the values of the cross section obtained from radio data for the model with $M_{\chi}=9 \mathrm{GeV}$, while they are not compatible for $M_{\chi}=500 \mathrm{GeV}$, and they are only marginally compatible for $M_{\chi}=60 \mathrm{GeV}$ (for comparison, see the values reported in the caption of Fig. 2). For $M_{\chi}=60 \mathrm{GeV}$, the comparison with the Fig. 4 is only apparently contradictory, since in Fig. 4 the Fermi upper limit does not appear to be exceeded, whereas from Table 3 we can see that the upper limit on the cross-section imposed by the gamma-ray limits is lower than the value required by radio data. This can be explained by considering that in the Fig. 4 the differential fluxes are represented, while in the Table 3 the integrated fluxes for $E>100 \mathrm{MeV}$ were used.

We also considered the predictions of the various DM models by using a B-field of Coma that has the intensity and radial 

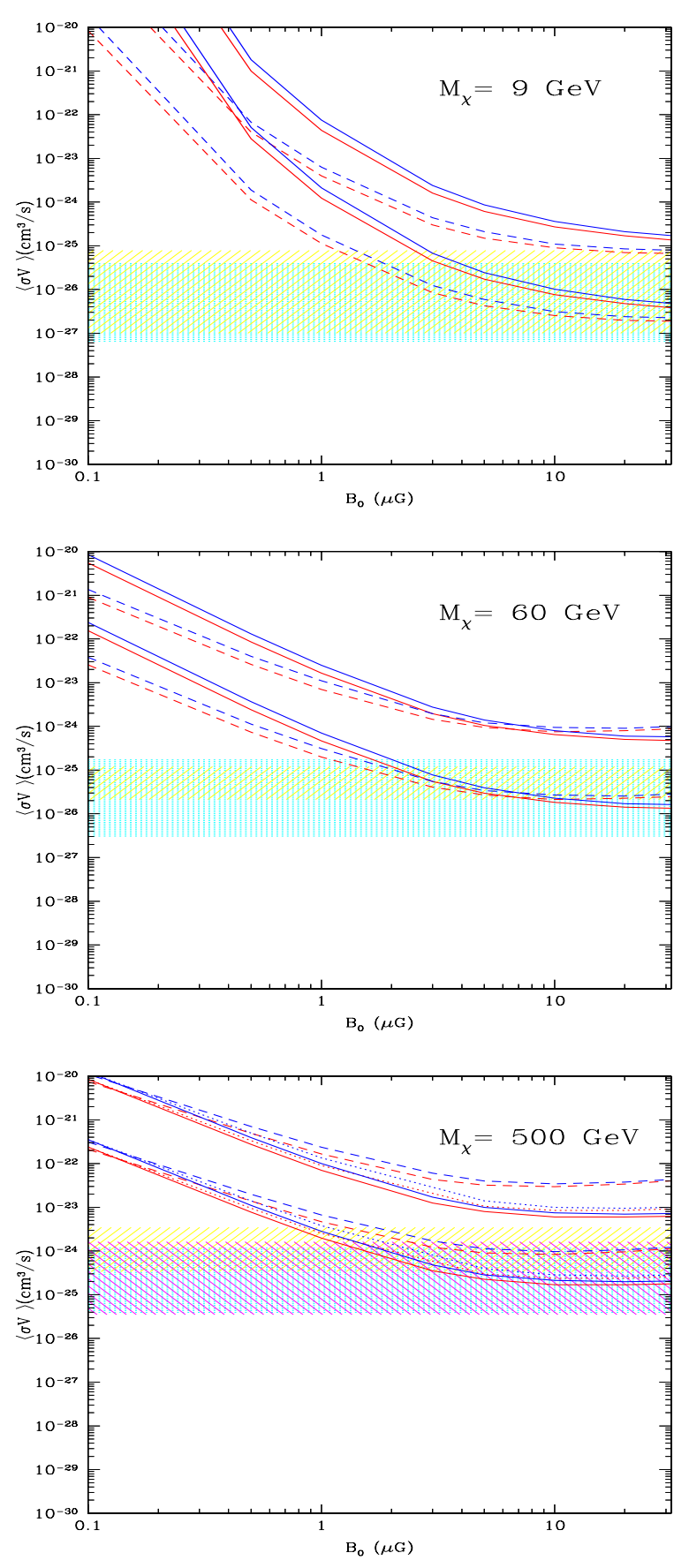

Fig. 6. We show the constraints set by the radio halo spectrum of Coma on the annihilation cross section for the DM models considered in this paper. Upper, middle, and lower panel refer to the 9,60 and $500 \mathrm{GeV}$ neutralino mass, respectively. We consider the case of an NFW DM profile with $b \bar{b}$ (solid red curve) and $\tau^{ \pm}$(dashed red curve), and the case for a cored DM profile with $b \bar{b}$ (solid blue curve) and $\tau^{ \pm}$(dashed blue curve). The lower panel also contains the cases of $\mathrm{W}^{ \pm}$composition with NFW (dotted red curve) and cored DM profile (dotted blue curve). The upper curve bundles refer to a smooth DM profile, while the lower curve bundles refer to the case where substructures are considered as in Fig. 1. The shaded bands in this figure represent the upper limit to the annihilation cross-section for the DM models derived combining the limits coming from a multi-messenger analysis (see text for details). The results are obtained using $B(r) \propto n_{\mathrm{th}}(r)$.

profile suggested by recent Faraday rotation measures (Bonafede et al. 2010, see Fig. 3). In this case, the DM model with $60 \mathrm{GeV}$ and $b \bar{b}$ composition and the model with $9 \mathrm{GeV}$ and $\tau^{ \pm}$composition would produce a radio halo spectrum with a shape that is still consistent with the observed one. The value of the annihilation cross-section required in the $60 \mathrm{GeV}, b \bar{b}$ case is $\sigma V=1.0 \times 10^{-24} \mathrm{~cm}^{3} \mathrm{~s}^{-1}$, while in the $9 \mathrm{GeV}, \tau^{ \pm}$case the crosssection required is $\sigma V=1.4 \times 10^{-25} \mathrm{~cm}^{3} \mathrm{~s}^{-1}$.

However, in order to have a DM-induced heating rate lower than the bremsstrahlung cooling rate of the intracluster gas at the cluster centre, the annihilation cross sections worked out here must be reduced by a factor $\sim 10^{7}-10^{12}$ for $M_{\chi}$ from 9 to $500 \mathrm{GeV}$, if we assume an NFW density profile.

This problem is, however, not present for a neutralino mass of $9 \mathrm{GeV}$ (with both $b \bar{b}$ and $\tau^{ \pm}$compositions) and $60 \mathrm{GeV}$ and composition $b \bar{b}$ if DM density has a cored profile (see Fig. 5), while it still holds for the $60 \mathrm{GeV}$ mass with composition $\tau^{ \pm}$and for all neutralino compositions in the $500 \mathrm{GeV}$ mass model.

The high-mass DM model with $M_{\chi}=500 \mathrm{GeV}$, whose SED is normalized to fit the Coma radio-halo data with a very low magnetic field value ( $B_{0} \approx 0.01 \mu \mathrm{G}$ for the $b \bar{b}$ composition), is inconsistent with the multi-frequency SED because it exceeds by large factors the EUV, soft X-ray, hard X-ray, and gammaray (in the energy range of Fermi) upper limits (see Fig. 4). For this high-mass DM model, also the $\tau^{ \pm}$and $W^{ \pm}$cases narrowly fail to fit the Coma radio-halo spectrum at low frequency below $\sim 400 \mathrm{MHz}$ (see Fig. 2).

We finally note that the high-energy HESS gamma-ray limit is not relevant in any of the DM models considered in this paper, because in all the cases the threshold energy of the HESS upper limits is higher than the neutralino masses considered here.

As a final remark, we stress that the DM candidates with $M_{\chi}=9 \mathrm{GeV}$ and $M_{\chi}=60 \mathrm{GeV}$ - those that best fit Coma astrophysical data - are too light to accommodate the PAMELA positron fraction (Adriani et al. 2009) or the electron-pluspositron flux seen by Fermi-LAT (Abdo et al. 2009) and HESS (Aharonian et al. 2008, 2009b).

In conclusion, the possibility of interpreting the origin of non-thermal phenomena in galaxy clusters with DM annihilation scenarios would require a low- or intermediate-neutralino mass and a cored DM density profile. If we then consider the multimessenger constraints to the neutralino annihilation crosssection, it turns out that these scenarios would be excluded as well unless we introduce a substantial boost factor that reproduces the presence of DM substructures.

Acknowledgements. M.P. acknowledges a grant from Fundação para a Ciência e Tecnologia (Ministério da Ciência, Tecnologia e Ensino Superior). L.P. thanks the University of Trento for hospitality.

\section{References}

Abdo, A. A., Ackermann, M., Ajello, M., et al. 2009, Phys. Rev. Lett., 102, 181101

Abdo, A. A., Ackermann, M., Ajello, M., et al. 2010, ApJ, 712, 147

Adriani, O., Barbarino, G. C., Bazilevskaya, G. A., et al. 2009, Nature, 458, 607

Aharonian, F., Akhperjanian, A. G., Barres de Almeida, U., et al. 2008, Phys. Rev. Lett., 101, 261104

Aharonian, F., Akhperjanian, A. G., Anton, G., et al. 2009a, A\&A, 502, 437

Aharonian, F., Akhperjanian, A. G., Anton, G., et al. 2009b, A\&A, 508, 561

Ahmed, Z., Akerib, D. S., Arrenberg, S., et al. 2009, [arXiv: 0912 .3592v1]

Baltz, E. A., \& Wai, L. 2004, Phys. Rev. D, 70, 023512

Bergstrom, L. 2000, Rept. Prog. Phys., 63, 793

Bertone, G., Hooper, D., \& Silk, J. 2005, Phys. Rep. 405, 279

Bertone, G., Cirelli, M., Strumia, A., \& Taoso, M., 2009, JCAP, 0903, 009

Blasi, P., \& Colafrancesco, S. 1999, Astropart. Phys., 12, 169

Bonafede, A., Feretti, L., Murgia, M., et al. 2010, A\&A, 513, A30

Bottino, A., Fornengo, N., \& Scopel, S. 2003, Phys. Rev. D, 67, 063519

Briel, U. G., Henry, J. P., \& Böhringer, H., 1992, A\&A, 259, L31 
Bringmann, T. 2009, New J. Phys., 11, 105027

Brunetti, G., Blasi, P., Cassano, R., \& Gabici, S. 2004, MNRAS, 350, 1174

Bullock, J. S., Kolatt, T. S., Sigad, Y., et al. 2001, MNRAS, 321, 559

Buote, D. 2001, ApJ, 553, 15

Carilli, C. L., \& Taylor, G. B. 2002, ARA\&A, 40, 319

Catena, R., Fornengo, N., Pato, M., Pieri, L., \& Masiero, A. 2009, [arXiv:0912.4421]

Celotti, A., Ghisellini, G., \& Chiaberge, M. 2001, MNRAS, 321, L1

Cen, R., \& Ostriker, J. P. 1999, ApJ, 514, 1

Cirelli, M., Kadastik, M., Raidal, M., \& Strumia, A., 2009, Nucl. Phys. B, 813, 1

Colafrancesco, S., \& Blasi, P. 1998, Astropart. Phys., 9, 227

Colafrancesco, S., \& Giordano, F. 2007, A\&A, 466, 421

Colafrancesco, S., \& Marchegiani, P. 2009, A\&A, 502, 711

Colafrancesco, S., \& Mele, B. 2001, ApJ, 562, 24

Colafrancesco, S., Marchegiani, P., \& Perola, G. C. 2005, A\&A, 443, 1

Colafrancesco, S., Profumo, P., \& Ullio, P. 2006, A\&A, 455, 21

Deiss, B. M., Reich, W., Lesch, H., \& Wielebinski, R. 1997, A\&A, 321, 55

Dennison, B. 1980, ApJ, 239, L93

Diego, J. M., \& Partridge, B. 2010, MNRAS, 402, 1179

Diemand, J., Kuhlen, M., Madau, P., et al. 2008, Nature, 454, 735

Donato, F., Fornengo, N., Maurin, D., \& Salati, P. 2004, Phys. Rev. D, 69, 063501

Durret, F., Kaastra, J. S., Nevalainen, J., Ohashi, T., \& Werner, N. 2008, Space Sci. Rev., 134, 51

Ellis, J. R., et al. 2005, Phys. Rev., D71, 095007

Ensslin, T., \& Biermann, P. L. 1998, A\&A, 330, 90

Farnier, C. 2009, talk given at Fermi Symposium Washington November 2009, web site: http://fermi.gsfc.nasa.gov/science/symposium/2009/ abs/cfarnier.html

Feretti, L., \& Giovannini, G. 2008, Lect. Notes Phys., 740, 143

Fitzpatrick, A. L., Hooper, D., \& Zurek, K. M. 2010 [arXiv: 1003.0014]

Fornengo, N., Pieri, L., \& Scopel, S. 2004, Phys. Rev. D, 70, 103529

Fusco-Fermiano, R., dal Fiume, D., Feretti, L. et al. 1999, ApJ, 513, L21

Fusco-Femiano, R., Orlandini, M., Brunetti, G., et al. 2004, ApJ, 602, L73

Geller, M. J., Diaferio, A., \& Kurtz, M. J. 1999, ApJ, 517, L23

Ghisellini, G., \& Celotti, A. 2001, in Issues of unifications of AGNs, ed. R. Maiolino, A. Marconi, \& N. Nagar [arXiv: astro-ph/0108110]
Giovannini, G., Feretti, L., Venturi, T., Kim, K.-T., \& Kronberg, P. P. 1993, ApJ, 406, 399

Gondolo, P., Edsjo, J., Ullio, P., et al. 2004, JCAP, 0407, 008

Green, A. M., Hofmann, S., \& Schwarz, D. J. 2005, JCAP, 0508, 003 [arXiv: astro-ph/0503387]

Hwang, C.-Y. 1997, Science, 278, 1917

Jaffe, W. J. 1977, ApJ, 212, 1

Kaastra, J. S., Lieu, R., Mittaz, J. P. D., et al. 1999, ApJ, 519, L119

Kim, K. T., Kronberg, P. P., Dewdney, P. E., \& Landecker, T. L. 1990, ApJ, 355, 29

Komatsu, E. et al. 2010, ApJS, accepted [arXiv: 1001.4538]

Kubo, J. M., Stebbins, A., Annis, J., et al. 2007, ApJ, 671, 1466

Lagana, T. F., de Souza, R. S., \& Keller, G. R. 2010, A\&A, 510, A76

Lieu, R., Mittaz, J. P. D., Bowyer, S., et al. 1996, Science, 274, 1335

Lieu, R., Ip, W.-H., Axford, W.-I., \& Bonamente, M. 1999, ApJ, 510, L25

Lieu, R., Mittaz, J. P. D., \& Zhang, S. N. 2006, ApJ, 648, 176

Lokas, E. L., \& Mamon, G. A. 2003, MNRAS, 343, 401

Lueker, M., Lueker, M., Reichardt, C. L., Schaffer, K. K., et al. 2010, ApJ, 719, 1045

Marchegiani, P., Perola, G. C., \& Colafrancesco, S. 2007, A\&A, 465, 41

Million, E. T., \& Allen, S. W. 2009, MNRAS, 399, 1307

Mittaz, J. P. D., Lieu, R., \& Lockman, F. J. 1998, ApJ, 498, L17.

Mori, M. 2009, 2009 Fermi Symposium, eConf Proc. C091122, [arXiv: 0912 . 3346]

Nath, B. B., Madau, P., \& Silk, J. 2006, MNRAS, 366, L35

Pato, M., Pieri, L., \& Bertone, G. 2009, Phys. Rev. D, 80, 103510

Pieri, L., Lavalle, J., Bertone, G., \& Branchini, E. 2009, [arXiv: 0908.0195]

Prokhorov, D. A. 2009, A\&A, 508, 59

Reimer, O., Pohl, M., Sreekumar, P., \& Mattox, J. R. 2003, ApJ, 588, 155

Rephaeli, Y. 1979, ApJ, 227, 364

Rephaeli, Y., Gruber, D., \& Blanco, P. 1999, ApJ, 511, L21

Rossetti, M., \& Molendi, S. 2004, A\&A, 414, L41

Roszkowksi, L., Ruiz de Austri, R., Trotta, R., et al. 2007, JHEP, 07, 075

Sarazin, C. L., \& Lieu, R. 1998, ApJ, 494, L177

Springel, V., Wang, J., Vogelsberger, M., et al. 2008, MNRAS, 391, 1685

Thierbach, M., Klein, U., \& Wielebinski, R. 2003, A\&A, 397, 53

Völk, H. J., Aharonian, F. A., \& Breitschwerdt, D. 1996, Space Sci. Rev., 75, 279 